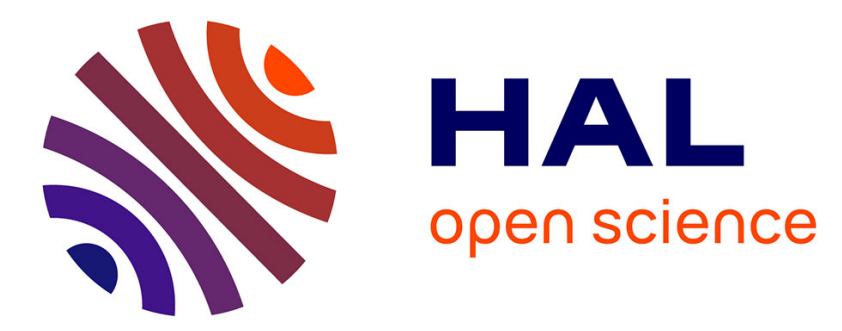

\title{
Tunable dual-mode DFB laser for millimetre-wave signal generation
}

\author{
S. Ginestar, F. van Dijk, A. Accard, F. Poingt, F. Pommereau, L. Le \\ Gouezigou, O. Le Gouezigou, F. Lelarge, B. Rousseau, J. Landreau, et al.
}

\section{- To cite this version:}

S. Ginestar, F. van Dijk, A. Accard, F. Poingt, F. Pommereau, et al.. Tunable dual-mode DFB laser for millimetre-wave signal generation. European Physical Journal: Applied Physics, 2011, 53 (3), pp.33609-1-5. 10.1051/epjap/2011100065 . hal-00672783

\section{HAL Id: hal-00672783 \\ https://hal.science/hal-00672783}

Submitted on 22 Feb 2012

HAL is a multi-disciplinary open access archive for the deposit and dissemination of scientific research documents, whether they are published or not. The documents may come from teaching and research institutions in France or abroad, or from public or private research centers.
L'archive ouverte pluridisciplinaire HAL, est destinée au dépôt et à la diffusion de documents scientifiques de niveau recherche, publiés ou non, émanant des établissements d'enseignement et de recherche français ou étrangers, des laboratoires publics ou privés. 


\title{
Tunable dual-mode DFB laser for millimetre-wave signal generation
}

\author{
Stéphane Ginestar* (1), Frédéric van Dijk (2), Alain Accard (2), Francis Poingt (2), Frédéric \\ Pommereau (2), Lionel Le Gouezigou (2), Odile Le Gouezigou (2), François Lelarge (2), \\ Benjamin Rousseau (2), Jean Landreau (2), Jean-Pierre Vilcot (1), \\ Guang-Hua Duan (2)
}

(1) Institut d'Electronique, de Microélectronique et de Nanotechnologie, UMR 8520, Université Lille 1, Avenue Poincaré, BP 60069, 59652 Villeneuve d'Ascq cedex, FRANCE

(2) Alcatel-Thales III-V Lab, Route de Nozay, 91460 Marcoussis FRANCE

*: Tel: +33 320197 991, Fax: +33 320197 966, Email: stephane.ginestar@iemn.univ-lille1.fr, frederic.vandijk@3-5lab.fr

\begin{abstract}
Highly compact dual-mode semiconductor laser sources get more and more attention in different application fields such as radar, security and personal communication systems. When the two generated wavelengths are detected within the same photodetector, an electrical signal which frequency is the difference between the frequencies of the two optical modes will be issued. We report on an integrated semiconductor device which is composed of two in-line DFB sections that are using the same optical waveguide structure. The so generated dual-mode spectrum can be adjusted first, by the difference in the grating parameters of each DFB section and second, by their respective driving current. We will report on the characterisation of such a device focusing on tunability and linewidth aspects that are of prime importance in above mentioned applications.
\end{abstract}

PACS: 42.55.Px, 07.57.Hm

\section{Introduction}

Millimetre-wave frequency range attracts more and more application fields such as radar, security and communication systems. As an example, short range high data rate Wireless Personal Area Networks (WPANs) got a recent standard $802.15 .3 \mathrm{C}$ [1] that allows specifying system and device characteristics in the $59-66 \mathrm{GHz}$ frequency range for short-range multi-gigabits wireless transmission systems. Radio over fibre (RoF) techniques and related devices are then key technologies for the design of future wireless access networks since fibre provides a smooth interconnection medium between all the different millimetre wave units (remote antenna units: RAUs) by its low attenuation, between $0.2 \mathrm{~dB} / \mathrm{km}$ and $0.5 \mathrm{~dB} / \mathrm{km}$, its easiness of installation and its low installation and maintenance costs [2]. In this paper, we will use this application field as demonstrator but one has to keep in mind that the dual-mode source that is reported here can be used in any other application field by the adjustment of the frequency spacing between the two generated optical tones, as it will be shown.

The basic implementation of the optical generation of a micro- or millimetre-wave signal by a dual-mode source is shown in Figure 1. The two optical modes which wavelengths, $\lambda_{1}$ and $\lambda_{2}$, are separated by the value corresponding to the millimetre-wave frequency, $f$, are launched into the receiver where a high bandwidth photodiode delivers the millimetre-wave frequency via a heterodyne detection scheme [3].

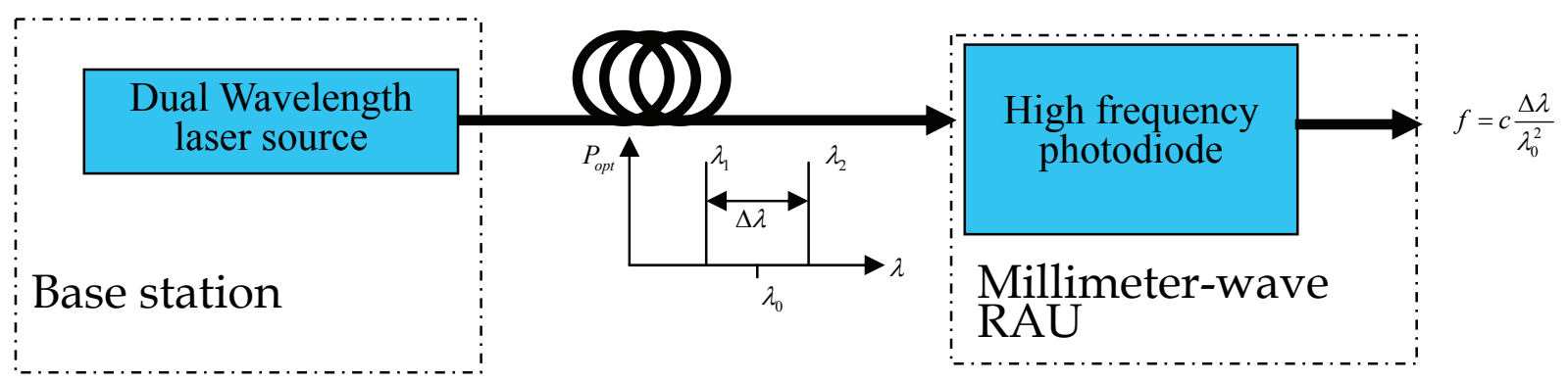

Figure 1: Details of optical millimetre-wave generation scheme 
Several processes allow generating this dual-tone optical signal; their concept has been reported ten to fifteen years ago. Some require an external microwave source such the implementations using an external MachZehnder modulator biased at its extinction voltage [4] or the use of two separate laser chips combined under master-slave arrangement [5] and others did not such a single laser chip generating two wavelengths [6]. All these configurations allow more or less the tunability of the spacing between the two optical modes and so the millimetre-wave frequency that is generated. Other solutions such using mode-locked lasers [7] are also compact ones but do not afford such tunability. In order to have a compact tunable source, we focus here on a single chip device generating two optical tones. It uses two DFB lasers that are combined within the same optical waveguide structure leading so to an in-line arrangement and a single optical output.

This scheme allows generating millimetre-wave signals on a huge frequency range from some GHz up to several THz. This frequency range can be coarsely adjusted during the fabrication step via the grating pitch value of each DFB section and finely tuned during operation via the driving current of each of these two sections.

We report here the design and performance of such a laser structure. We particularly focus on the characterization of the tunability and linewidth of both optical and electrical tones since they are of prime importance in radio over fibre communication applications.

\section{Design and fabrication of the laser structure}

The laser structure is based on a ridge structure [8]. The active layer is composed of InGaAsP quantum wells (6) and barriers. Figure 2 shows a schematic view of the dual-DFB structure. It is composed of two $525 \mu \mathrm{m}$-long DFB sections. One phase section is located between these two. An unbiased $1200 \mu \mathrm{m}$-long section is used at one end of the laser as an absorbing layer and act so as a perfect anti-reflection coating.

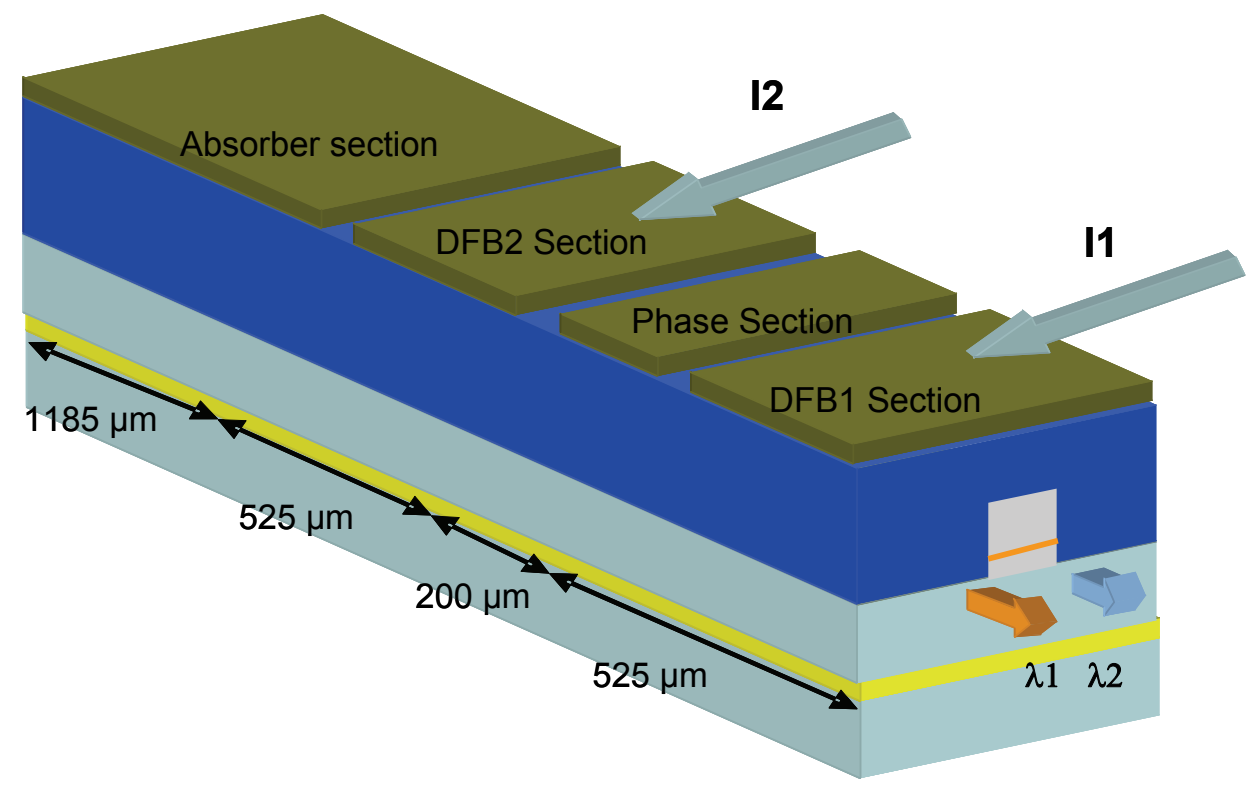

Figure 2: Schematic view of the dual-DFB laser structure

As previously mentioned, each DFB section, DFB1 and DFB2, has a different grating pitch, respectively $\Lambda_{1}$ and $\Lambda_{2}$. These sections incorporate a $\lambda / 4$ phase shift and their emission wavelength is then defined as:

$$
\left.\lambda_{i}=2 n_{\text {eff }} \Lambda_{i} \text { (with } i=1 \text { or } 2\right)
$$

with $\Lambda_{i}$, the grating pitch of DFB section $i$ and $\mathrm{n}_{\text {eff }}$, the effective index of the guided optical mode.

The microwave or millimetre-wave frequency that will be generated is related to the difference between the two optical frequencies and so between the two grating pitches.

$$
F=c \frac{\Delta \lambda}{\lambda^{2}}=\frac{2 c n_{\text {eff }}}{\lambda^{2}}\left|\Lambda_{1}-\Lambda_{2}\right|
$$

With c: light velocity

$\Delta \lambda$ : wavelength difference between the two emission wavelengths $\left(\left|\lambda_{1}-\lambda_{2}\right|\right)$ 
Taking into account an emission wavelength $(\lambda)$ in the $1.55 \mu \mathrm{m}$ range, Eq. 2 leads to a frequency spacing between the two optical modes around $400 \mathrm{GHz}$ for a difference of grating pitch of $3 \mathrm{~nm}$. As above mentioned, this can be seen as a coarse tuning of the millimetre-wave frequency range that can be obtained. We used e-beam lithography to define these gratings. This allows achieving this slight grating pitch variation within the same lithography step.

\section{Tunability}

Besides the frequency difference between the two modes that is obtained by the grating pitch difference between the two sections, fine tuning of this difference can be achieved by modifying the injection current (and so the material index) of each section. The threshold current of each section is $20 \mathrm{~mA}$. We fixed the bias of one section to $50 \mathrm{~mA}$ and tuned the bias of the other section up to $250 \mathrm{~mA}$. Figure 3 shows the output optical spectra of a laser with a $0.3 \mathrm{~nm}$ difference in the grating pitch values value for 3 different combinations. The rough observation of this figure shows two different behaviours with the bias current difference: the first one is actually the frequency difference between the two tones and the second one is the overall shift of the spectrum towards higher wavelength range which is due to the heating of the laser chip (no cooler was used) when increasing the bias current of the second section.

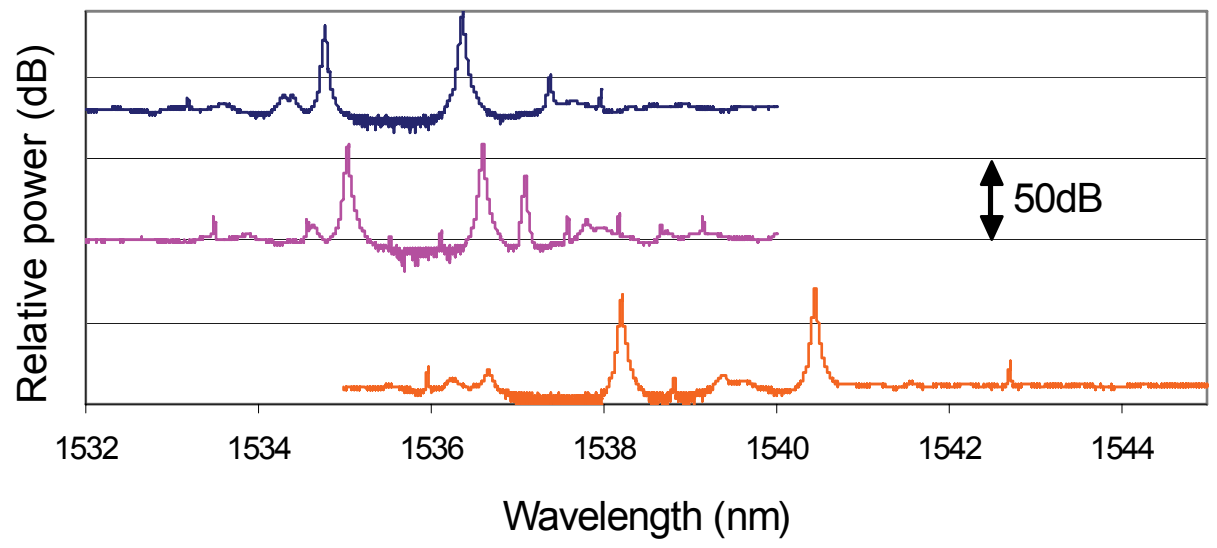

Figure 3: Output optical spectra of the dual mode laser: $\mathrm{I} 1=50 \mathrm{~mA} ; \mathrm{I} 2=60 \mathrm{~mA}$ (upper trace), $100 \mathrm{~mA}$ (middle trace) and $250 \mathrm{~mA}$ (lower trace)

Figure 4a shows the power difference between the two optical tones versus the bias current of the second section; the bias current of the first section is kept unchanged $(50 \mathrm{~mA})$. The second section bias current is varying from $50 \mathrm{~mA}$ to $300 \mathrm{~mA}$.

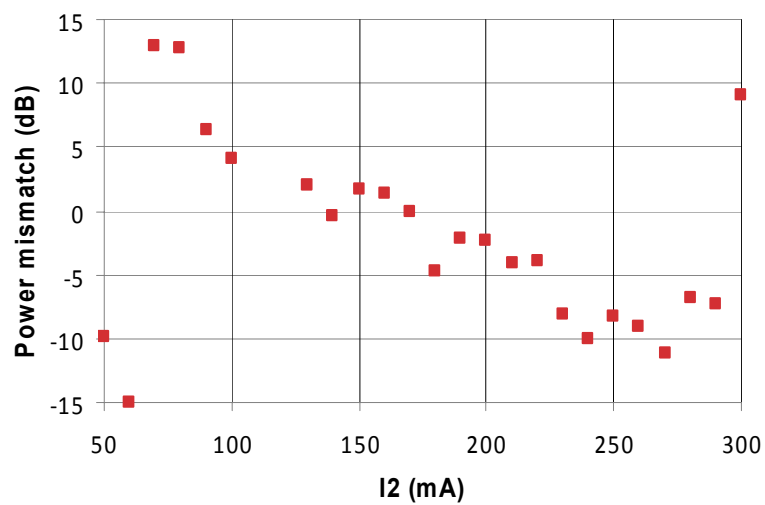

a)

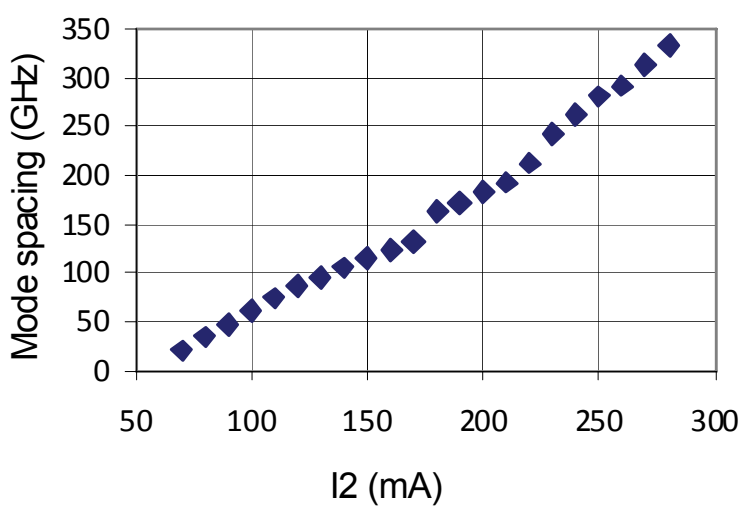

b)

Figure 4: Characteristics of the two-tone signal a): relative power difference, b): frequency spacing In both cases, the current of the first section (I1) is fixed at $50 \mathrm{~mA}$ and the current of the second section (I2) is tuned between 50 and $300 \mathrm{~mA}$.

First, we can notice that for almost the same value of driving current in both sections, i.e. $50 \mathrm{~mA}$, a rather large difference is obtained on their output power; in a first approach, this could somehow be surprising. 
Looking more in details, this can be related to the stop band effect of one DFB section to the other. The stop band width of a DFB section, depending on the laser structure, can reach hundred of GHz. That is to say that, as both DFB sections are in-line, if the emission wavelength of one DFB section falls within the stop band of the other DFB section, a decrease of output power occurs. This is clearly observed for I2 values between 50 and roughly $100 \mathrm{~mA}$ where a large mismatch in the output power of the two tones is observed. As a second observation item, it can be noted that both modes get almost an equivalent output power for I2 between 120 and roughly $200 \mathrm{~mA}$. When $\mathrm{I} 2$ is greater than $200 \mathrm{~mA}$, the mismatch between the output powers of the two modes increases again but it is expectable due to the large mismatch in the driving current of both sections.

Figure $4 \mathrm{~b}$ shows the frequency difference between the two optical tones versus the bias current of the second section; the bias current of the first section is kept unchanged (50 mA) (same conditions as above); the frequency difference is between $20 \mathrm{GHz}$ and $300 \mathrm{GHz}$.

Some concluding remarks can so be made concerning the tunability of this dual-mode DFB laser structure: - a rather large tunability of the mode spacing can be achieved by the tuning of the current difference between the two sections,

- inherently, the output power mismatch between the two modes is directly dependent on the current difference between the two sections,

- superimposed to this output power difference related to the difference in the driving current of both sections, the stop band effect of one DFB section to the emission power of the other DFB section can give rise to supplementary output power mismatch. This phenomenon is essentially valid when both emission wavelengths are close from each other, e.g. for a frequency spacing below $100 \mathrm{GHz}$, which is the mean value of the stop band width of a DFB section.

\section{Optical signal linewidth}

For most of applications, particularly for telecommunications applications, the linewidth of the electrical carrier signal is of prime importance since its noise behaviour will directly impact on the communication performance. Usually, the linewidth of the electrical signal resulting from the beating of two optical tones in a photodetector is told to be equal to the double of the mean value of the optical linewidths [11].

So, in a first step, we measured the mean value of optical linewidth. We used the self-heterodyne method which has been described in [11]. The laser output signal is divided in two parts. One part is sent into an acoustooptic modulator which shifts all optical frequencies by some tens of $\mathrm{MHz}$; in our case the driving frequency of this modulator (and so the frequency shift) is $80 \mathrm{MHz}$. The other part is sent to a long optical fibre in order to remove the coherence between the two lights beams at their recombination point and so any interference pattern that could occur. The beating note within the detector, which is centred at the frequency of the acousto-optic modulator driving signal is then analysed by a low frequency spectrum analyser.

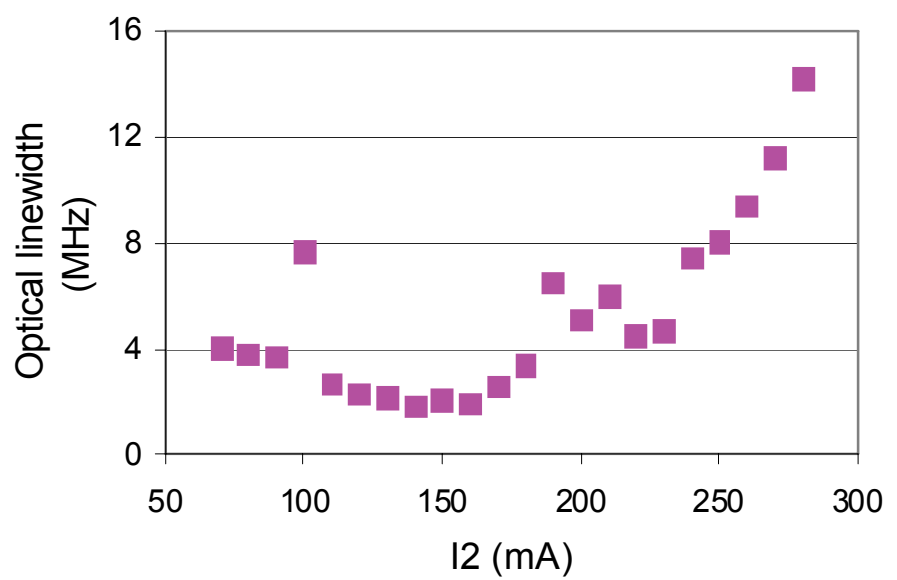

Figure 5: Mean value of the signal linewidth of the two optical tones

Taking into account that the optical spectrum has a lorentzian form, the optical linewidth is obtained from the electrical tone measurement by dividing its linewidth by 2 . Since both optical tones have been measured simultaneously, the obtained value is so a mean value of the two optical tone linewidths as far as their power is identical (otherwise, the obtained result shall be weighted by the relative power level of these two signals). Figure 5 shows the so obtained optical signal linewidth versus the bias current of the second section, the current of the first section is always kept fixed at $50 \mathrm{~mA}$. The linewidth varies from 4 to $14 \mathrm{MHz}$ that corresponds to the one that is commonly obtained for such ridge MQW laser structures. 


\section{Electrical signal linewidth}

The set-up is here almost simple. The output signal of the laser is sent to a high bandwidth $(50 \mathrm{GHz})$ photodiode connected to a spectrum analyser. The electrical linewidth is directly measured.

Measurements have been made for the bias current of the second section varying up to $110 \mathrm{~mA}$ (first section bias current still fixed at $50 \mathrm{~mA}$ ). Above this value, the frequency of the electrical beating note is too high to get relevant signal at the photoreceiver output. Figure 6 shows these results and the corresponding optical tone linewidth that has been obtained from optical measurements (see $\S 4$ ).

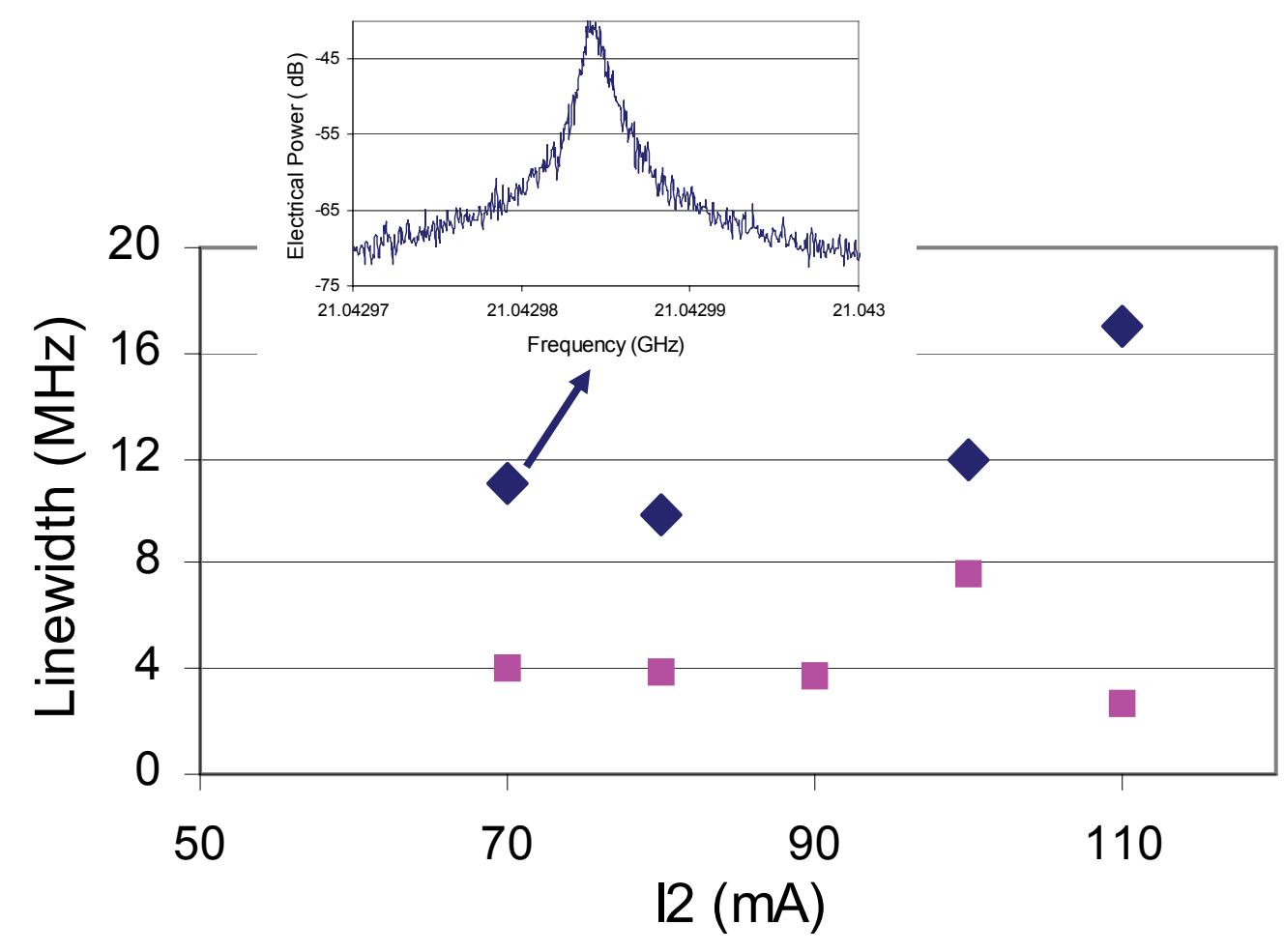

Figure 6: Comparison between optical linewidth (square dots) measured in $\S 4$ and electrical linewidth (diamond dot). In insert, a typical spectrum obtained, in that case, for a bias current of section 1 and 2 equal to $50 \mathrm{~mA}$ and $70 \mathrm{~mA}$, respectively.

The range of variation for the electrical linewidth is comprised between $10 \mathrm{MHz}$ and $18 \mathrm{MHz}$ which is a little bit more than the factor of 2 that is commonly used.

For applications as security, sensing, such a linewidth would be adequate. For metrology or high data rates communications, a lower linewidth, in the order of $1 \mathrm{MHz}$, would be expectable. Structures using quantum dash based active materials are being fabricated in order to fulfil this requirement.

\section{Conclusion}

A dual-mode DFB laser with a frequency difference between its two emission wavelengths varying from 30 $\mathrm{GHz}$ to $300 \mathrm{GHz}$ has been fabricated; it consists of an in-line arrangement of $2 \mathrm{DFB}$ sections. In the reported case, the grating pitch difference between the two DFB sections is $0.3 \mathrm{~nm}$.

The frequency tuning is obtained by tuning the bias current of one DFB section respectively to the second one. Although a wide tuning range can be so obtained, the limit of the structure is then the power unbalance between the two generated modes. This power unbalance has two different origins depending on device conception and working conditions. First, it is obvious that, as these two DFB sections, except by their grating pitch value, are identical, higher the driving current unbalance between the two sections will be, higher the output power unbalance will be. Second, as both DFB sections are in-line, the mode of one section propagates also through the other; if the emission wavelength of one of the DFB sections is located within the stop band of the other DFB section, a strong attenuation occurs then. So, even if wide tunability can be achieved, the optimum working conditions, i.e. equal power on the two output modes, is only obtained for equivalent biasing conditions on the two sections. That is to say that such a device is best suited for a two-tone generator which frequency spacing is well defined. In that case, the grating pitch difference performs this spacing definition, driving current tunability being then used as to balance the fabrication tolerance. Third, reaching small (some tens of $\mathrm{GHz}$ ) frequency spacing will forever be difficult since the stop band effect will be complex to overcome in that 
particular case. It is so best to recommend this device structure for frequency spacing higher than, let us say, $100 \mathrm{GHz}$ wich is the mean value of stop band width. For small frequency differences, a parallel arrangement of two DFB sections associated with a multi-mode interferometer coupler will definitely be a better solution, but more complex to implement.

It has been verified that the electrical signal linewidth issued from the beating of the two optical tones is a little bit more than 2 times the mean optical linewidth. An electrical signal linewidth around $10 \mathrm{MHz}$ has been obtained up to $50 \mathrm{GHz}$, which was the limit of our photodetector. The photogenerated electrical signal linewidth enables the use of such an optical generator for security or sensing applications. Telecommunications applications can also be investigated if the bit rate or the modulation scheme are not too high or too complex, respectively. In these later cases, the obtained linewidths could be too wide. Sticking on the same device configuration, the switch to a quantum dash based active layer shall decrease the optical signal linewidth and, consequently, the photogenerated electrical signal one.

\section{Acknowledgements}

This work has been supported by the European Commission under the FP6-IP-35317 "IPHOBAC" project (www.ist-iphobac.org).

\section{References}

[1] R Fisher, "60Ghz WPAN Standardization with IEEE 802.15.3C" Signal, Systems and Electronics, 2007, ISSSe07 international Symposium, July 30 2007-Aug. 2 2007, Page 103-105.

[2] D. Wake, "Radio over Fiber Systems for Mobile Applications" in Radio over Fiber Technologies for Mobile Communications Networks”, H. Al-Raweshidy, and S. Komaki, ed. (Artech House, Inc, USA, 2002).

[3] D. Wake et al., "Optical generation of millimeter-wave signals for fiber-radio systems using a dual-mode semiconductor laser", IEEE Trans. Micr. Theo. Techn. 43, 2270 (1995).

[4] J.J. O'Reilly et al., "Optical generation of very narrow linewidth millimeter-wave signals", Electron. Lett. 28 , 6309 (1992)

[5] L. Noel et al., "Optical millimetre-wave generation technique with high efficiency, purity and stability", Electron. Lett. 32, 1997 (1996).

[6] C. R. Lima et al., "Compact optical millimeter-wave source using a dual-mode semiconductor laser", Electron. Lett. 31, 364 (1995).

[7] D. Novak et al., "Locking range of a hybrid mode-locked monolithic DBR semiconductor laser at millimetre-wave frequencies", IEEE Micr. And Guided Waves Lett. 6, 320 (1996).

[8] F. Lelarge et al. "Processing of InP-based shallow ridge laser waveguides using a HBr ICP plasma"in Proceedings of International Conference on Indium Phosphide and Related Materials (IPRM) 2006 , Princeton, edited by IEEE, p. 127.

[8] Al-Mumin et al., "Optical generation and side band injection locking of tunable 11-120GHz microwave/millimeter signals“, Electron. Lett. 36, 1547 (2000)

[9] B. Sartorius et al., "12-64Ghz continuous frequency tuning in self-pulsating $1.55 \mu \mathrm{m}$ multi quantumwell DFB lasers“, IEEE Journ. of Sel. Top. in Quant. Elect. 1, 535 (1995) .

[11] T. Okoshi et al., "Novel method for high resolution measurement of laser output spectrum", Electron. Lett. 16, 630 (1980). 